\title{
NIGELLA SATIVA OIL AS AN AGENT IN ENHANCING IMMUNITY AGAINST COVID-19
}

\author{
D.M. Abdulah
}

The outbreak of coronavirus disease 2019 (COVID-19) is continuing since its last occurrence in December 2019 in Wuhan, China. The disease has been spread broadly in China and several other countries and territories, including Iraqi Kurdistan (1). The World Health Organization (WHO) has recently declared COVID-19 a public health emergency of international concern (2).

The patients diagnosed with COVID-19 have a fever on admission $(43.8 \%)$ and during hospitalization $(88.7 \%)$, followed by cough $(67.8 \%$ of patients). The laboratory findings have shown that lymphocytopenia is present on admission in $83.2 \%$ and thrombocytopenia in $36.2 \%$, and leukopenia in $33.7 \%$ of the patients. The elevated level of C-reactive protein was seen in most of the patients. The patients with severe disease had more prominent laboratory abnormalities, including lymphocytopenia and leukopenia, compared to those with the non-severe disease (3).

Currently, there is no vaccine or therapy for COVID19. It takes several months away to develop a vaccine or treatment for COVID-19. The health care forces are under high pressure globally through the overwhelming potential burden of illnesses and their adverse effects on health care works, such as the risk of infection. It is estimated that 3000 health care workers were infected by this virus in China; of these patients 22, health care workers died. Therefore, we need to find possible therapeutic agents to raise the immunity of the affected persons by COVID-19 against the virus.

Black seed (known as black cumin; Nigella sativa) is an annual flowering plant belonging to the family Ranunculaceae. It is a native of Southern Europe, North Africa, and Southwest Asia. Nigella sativa is cultivated in the Middle Eastern Mediterranean region, Southern Europe, Northern India, Pakistan, Syria, Turkey, Iran, and Saudi Arabia. The black cumin seeds have been used in Southeast Asian and Middle East countries to treat several diseases like asthma, bronchitis, rheumatism, and other inflammatory diseases. Nigella sativa is being used extensively due to its potential therapeutic properties. It has a broad function, such as immune-modulatory, antimicrobial, and anti-inflammatory properties (4).

Corresponding Author: Deldar Morad Abdulah, Community Health Unit, College of Nursing, University of Duhok-Iraqi Kurdistan, Email: deldarmorad@gmail.com, Phone: +9647507443319; ; ORCID: https: / / orcid.rg/0000-0002-8986-5793
Nigella sativa seeds have the following constituents; protein $(26.7 \%)$, fat $(28.5 \%)$, carbohydrates $(24.9 \%)$, crude fiber $(8.4 \%)$, and total ash $(4.8 \%)$. These seeds have a good amount of different vitamins and minerals such as $\mathrm{Cu}, \mathrm{P}, \mathrm{Zn}$, and $\mathrm{Fe}$ along with several active components; including thymoquinone (TQ) (30\%-48\%), thymohydroquinone, dithymoquinone (nigellone), p-cymene $(7 \%-15 \%)$, carvacrol $(6 \%-12 \%)$, 4-terpineol $(2 \%-7 \%)$, t-anethole $(1 \%-4 \%)$, sesquiterpene longifolene $(1 \%-8 \%), \alpha$-pinene, and thymol (4).

The immunomodulatory properties of Nigella sativa and its active ingredient, TQ in modulating cellular and humoral adaptive immune responses, were extensively reviewed (5). In an in vivo investigation, the oral administration of Nigella sativa oil was shown to improve lymphocyte count (such as lymphocyte proliferation) in the peripheral blood of streptozotocin (STZ)-induced diabetic hamsters substantially. The Experimental evidence recommends that N. Sativa extracts and TQ enable therapeutically to be used in the regulation of immune reactions in infectious and non-infectious conditions like allergy, autoimmunity, and cancer. Nigella sativa metabolic extract treatment improves the total white blood cell counts and increased spleen weight in BALB/c mice, suggesting the role of the immunomodulatory activity of N. Sativa seeds (6). Co-administration of N. Sativa (2.5\%) with oxytetracycline completely blocks a decrease in the leukocyte and lymphocyte through oxytetracycline and produced immunostimulant effects in pigeons indicating an immune-protective effect (7). A study reported complete recovery and sero-reversion of HIV infection following treatment with N. Sativa concoction $(60 \% \mathrm{~N}$. Sativa seeds and $40 \%$ honey) for 6 months in a 46-year old man with human immunodeficiency virus (HIV) (8).

A study examined the effectiveness of the modulatory effect of Nigella sativa on the selected $T$ cell subset in female patients with rheumatoid arthritis in a randomized, double-blinded placebo-controlled trial. The patients in the treatment group received two capsules containing NS oil extracts (each $500 \mathrm{mg}$ ). The substantial decline in the serum high-sensitivity C-reactive protein (hs-CRP) level and disease activity scores of 28 joints scores were found in the experimental arm. The significant reduction in CD8+ and an increase in 
CD4+CD25+ $\mathrm{T}$ cell percentage and the CD4+/CD8+ ratio was found in the treatment group (9).

A study reviewed the clinical uses of Nigella sativa and TQ to prevent and treat different diseases and morbidities in humans (10). The benefits of the black seed and TQ were reported in several diseases such as inflammatory and auto-immune disorders, and metabolic syndrome. The advantages were antimicrobial, anti-nociceptive, and anti-epileptic properties with no serious side effects. The Nigella sativa has been used in a double-blind randomized clinical trial in patients with type 2 diabetes mellitus (T2DM). The study showed that Nigella sativa oil as two capsules daily for 8 weeks is related to decrease in FBS, triglyceride, total cholesterol, low-density lipoprotein cholesterol, serum hs-CRP, malondialdehyde and increase in serum level of highdensity lipoprotein cholesterol in intervention group (11). The effectiveness of Nigella sativa oil to improve disease outcomes in hospitalized adult COVID-19 patients is under investigation still (12) or it has been suggested recently (13).

The patients with COVID-19 are presented to a significantly lower level of immunity, including lymphocytopenia, thrombocytopenia, and leukopenia. Therefore, Nigella sativa oil should be tested in the context of prevention and/or complementary treatment against COVID-19.

Sources of support: There was no funding to this study.

Acknowledgments: I would like to present my deep thanks to Mrs. Shireen Taha Saadullah for this suggestion.

\section{References}

1. Abdulah, DM, SSA Qazli, and SK Suleman. Response of the public to preventive measures of coronavirus infection in Iraqi Kurdistan. Disaster Medicine and Public Health Preparedness: 1-22.

2. World Health Organization. Coronavirus disease (COVID-19) outbreak. 2020; Available from: https: / www.who.int.

3. Guan, W-j, Z-y Ni, Y Hu, et al. Clinical characteristics of coronavirus disease 2019 in China. New England Journal of Medicine, 2020.

4. Srinivasan, K. Cumin (Cuminum cyminum) and black cumin (Nigella sativa) seeds: traditional uses, chemical constituents, and nutraceutical effects. Food quality and safety, 2018. 2(1): 1-16.

5. Majdalawieh, AF and MW Fayyad. Immunomodulatory and antiinflammatory action of Nigella sativa and thymoquinone: A comprehensive review. International immunopharmacology, 2015. 28(1): 295-304.

6. Ghonime, M, R Eldomany, A Abdelaziz, et al. Evaluation of immunomodulatory effect of three herbal plants growing in Egypt. Immunopharmacology and Immunotoxicology, 2011. 33(1): 141-5.

7. Abel-Salam, BK. Immunomodulatory effects of black seeds and garlic on alloxan-induced diabetes in albino rat. Allergologia et immunopathologia, 2012. 40(6): 336-40.

8. Onifade, AA, AP Jewell, and WA Adedeji. Nigella sativa concoction induced sustained seroreversion in HIV patient. African Journal of Traditional, Complementary and Alternative Medicines, 2013. 10(5): 332-5.

9. Kheirouri, S, V Hadi, and M Alizadeh. Immunomodulatory effect of Nigella sativa oil on $\mathrm{T}$ lymphocytes in patients with rheumatoid arthritis. Immunological investigations, 2016. 45(4): 271-83.

10. Tavakkoli, A, V Mahdian, BM Razavi, et al. Review on clinical trials of black seed (Nigella sativa) and its active constituent, thymoquinone. Journal of pharmacopuncture, 2017. 20(3): 179.

11. Kooshki, A, T Tofighiyan, N Rastgoo, et al. Effect of Nigella sativa oil supplement on risk factors for cardiovascular diseases in patients with type 2 diabetes mellitus. Phytotherapy Research, 2020.

12. Koshak, AE. Nigella Sativa in COVID-19. 2020; Available from: https:// clinicaltrials.gov/ct2/show/NCT04401202.

13. Rahman, MT. Potential Benefits of Combination of Nigella sativa and Zn Supplements to Treat COVID-19. Journal of Herbal Medicine, 2020: 100382. 